\title{
A experiência e atitudes de adolescentes frente à sexualidade ${ }^{\#}$ Experience and attitudes of teenagers facing sexuality
}

\author{
Fernanda Cristina Aguiar Lima* \\ Flávia Barbosa de Jesus** \\ Christine Baccarat de Godoy Martins*** \\ Solange Pires Salomé de Souza**** \\ Karla Fonseca de Matos****
}

\section{Resumo}

Frente ao evento da iniciação sexual precoce, gravidez e doenças sexualmente transmissíveis na adolescência, torna-se imprescindível conhecer como o adolescente vivencia sua sexualidade para que esses resultados possam orientar meIhor ações preventivas. Este estudo teve por objetivo conhecer a atitude dos adolescentes frente à sexualidade. Trata-se de estudo descritivo, com 499 alunos do primeiro ano do ensino médio, cujos dados foram coletados por questionário fechado e analisados pelo Epi Info. Foram pesquisados 190 meninos (38,1\%) e 309 meninas (61,9\%). Foi observado que há pouco diálogo sobre sexualidade no namoro, a iniciação sexual é mais precoce entre os meninos, a primeira relação sexual ocorre com o namorado (para as meninas) e sem compromisso de namoro (para os meninos). Possuem vida sexual ativa no momento $67,2 \%$ dos adolescentes, com frequência eventual, com 1 a 3 parceiros nos últimos três meses para as meninas $(72,3 \%)$, sendo que $48,1 \%$ dos meninos não se lembram. Declararam-se heterossexuais $71,7 \%$. Pôde-se concluir que se tornam relevantes práticas educativas quanto à sexualidade do adolescente, bem como o envolvimento da família para o diálogo e orientação.

Palavras-chave: Sexualidade. Comportamento do Adolescente. Saúde do Adolescente. Sexo sem Proteção. Enfermagem.

\begin{abstract}
Considering early sexual initiation, pregnancy and sexually transmitted diseases in adolescence, it is essential to know how the adolescent experiences his sexuality in order to better guide preventive actions. This study aimed at knowing the attitudes of adolescents regarding sexuality. This is a descriptive study, with 499 high school first-year students. Data were collected by closed questionnaire and analyzed using Epi Info. One hundred ninety boys (38.1\%) and 309 girls (61.9\%) were surveyed. It was observed that there is little dialogue about sexuality among partners, the sexual initiation happens earlier among boys and the first sexual relation happens with a boyfriend (for girls) and without any commitment (for boys). At the moment, $67.2 \%$ of the interviewed teenagers have an active sexual life with casual frequency of 1 to 3 partners for girls in the last three months $(72.3 \%)$, and $48.1 \%$ of the boys do not remember this information. $71.7 \%$ of the interviewed stated to be heterosexual. It was concluded that educational practices within the teenagers' sexuality and the involvement of the family in dialogue and guidance are important.
\end{abstract}

Keywords: Sexuality. Adolescent Behavior. Adolescent Health. Unsafe Sex. Nursing.

\# Pesquisa financiada pela FAPEMAT (Fundação de Amparo à Pesquisa de Mato Grosso). Processo n. 407104/2009.

* Enfermeira. Mestranda de Enfermagem pela Universidade Federal de Mato Grosso - UFMT, Cuiabá-MT, Brasil. E-mail: fer_nanda_lima@ hotmail.com

** Graduanda de Enfermagem pela Universidade Federal de Mato Grosso - UFMT, Cuiabá-MT, Brasil. E-mail: flavinha3004@hotmail.com

*** Enfermeira. Doutora em Saúde Pública pela Faculdade de Saúde Pública da USP-SP. Docente da Faculdade de Enfermagem da UFMT. Área Saúde da Criança e do Adolescente. Cuiabá-MT, Brasil. E-mail: leocris2001@terra.com.br

**** Enfermeira. Doutora em Enfermagem em Saúde Pública pela Faculdade de Enfermagem da USP, Ribeirão Preto-SP. Docente da Faculdade de Enfermagem da UFMT. Área Saúde da Criança e do Adolescente. Cuiabá-MT, Brasil. E-mail: solps@terra.com.br

***** Enfermeira. Mestranda em Enfermagem pela Universidade Federal de Mato Grosso, Faculdade de Enfermagem. Cuiabá-MT, Brasil. E-mail: karla.matos88@gmail.com

As autoras declaram não haver conflitos de interesse. 


\section{INTRODUÇÃO}

A adolescência é marcada por profundas mudanças físicas, sociais e psicoemocionais, o que representa um dos momentos mais vulneráveis do ciclo vital humano, ocorrendo novas formas de interação e inserção social, com manifestações peculiares de novos modos de pensar, sentir e comportar-se. O desenvolvimento da maturidade física e emocional, a inserção no mundo adulto e do trabalho e a descoberta da sexualidade tornam a adolescência um fenômeno bastante complexo ${ }^{1}$.

Entre as várias mudanças na adolescência, a mais conflituosa é a sexualidade, pois está relacionada a muitas crises e preocupações, que aparecem repletas de valores morais e preconceitos, vindos da família e sociedade, despertando situações-problema com os adolescentes ${ }^{2}$.

Em relação à iniciação sexual, observa-se que esta tem ocorrido cada vez mais cedo e que o número de parceiros tem aumentado significativamente nos últimos anos, entre os adolescentes ${ }^{3}$. Segundo as autoras, alguns fatores podem contribuir para essas mudanças, como a maior possibilidade de adiamento do convívio conjugal e maior abertura sexual nas últimas décadas.

Em Cuiabá, somente em setembro de 2009, as internações pelo Sistema Único de Saúde (SUS) decorrentes de gravidez, parto e puerpério representaram 29,4\% do total das internações entre jovens de 10 a 14 anos e 75,8\% entre os de 15 a 19 anos $^{4}$. Frente ao evento da iniciação sexual precoce, gravidez e doenças sexualmente transmissíveis (DST / AIDS) nessa faixa etária, torna-se imprescindível a orientação sexual no sentido da prevenção ${ }^{5}$. Para tanto, é preciso, primeiramente, conhecer como o adolescente tem vivenciado sua sexualidade, para que esses resultados possam orientar meIhor essas ações.

Nesse sentido, o presente estudo teve por finalidade conhecer a atitude dos adolescentes, do primeiro ano do ensino médio das escolas da rede pública estadual de ensino de Cuiabá-MT, frente à sexualidade, tendo por hipótese a existência de comportamento de risco entre adolescentes no que se refere à sexualidade.

\section{MÉTODO}

Trata-se de um estudo de corte transversal, com análise quantitativa, cuja população de estudo foi composta por 499 alunos do primeiro ano do ensino médio, sendo 190 meninos (38,1\%) e 309 meninas $(61,9 \%)$. Foi delimitado como critério de inclusão ter idade entre 10 e 19 anos, período que a Organização Mundial da Saúde (OMS) define como adolescência.

O estudo foi desenvolvido em 5 (cinco) escolas estaduais do município de Cuiabá-MT, previamente selecionadas em conjunto com a Secretaria de Estado de Educação - SEDUC, de forma aleatória, totalizando 22 turmas, com uma amostra de 499 alunos.

Para coleta dos dados, foi utilizado um questionário composto por 13 questões, sendo 9 fechadas e 4 abertas, aplicado à população de estudo pelas próprias pesquisadoras, auxiliadas por uma equipe de voluntários.

Os questionários foram entregues aos alunos para que respondessem às questões. Ao término, eram depositados em caixas lacradas, pelos próprios adolescentes, buscando, assim, aumentar a confiabilidade das respostas, uma vez que não era preciso se identificar nem mesmo entregar o instrumento respondido às pesquisadoras.

$\mathrm{O}$ estudo adotou as seguintes categorias de análise: com quem os adolescentes conversam sobre sexo; se existe diálogo sobre sexo dentro do atual relacionamento; a idade da primeira ejaculação / menstruação; se já teve relação sexual; com que idade teve a primeira relação sexual; com quem foi a primeira relação sexual; se houve pressão entre o(a) adolescente e o(a) parceira(a) para acontecer a primeira relação sexual; se utilizam algum método contraceptivo para evitar a gravidez e DST / AIDS; se atualmente possui vida sexual ativa; com que frequência tem relação sexual; qual a opção sexual do adolescente; a quantidade de parceiros que os adolescentes tiveram nos últimos três meses; se fazem uso de preservativo e com que frequência.

Os dados foram processados pelo programa Epi Info (versão 3.5.1) e analisados por meio de frequência absoluta e relativa. Foram realizadas algumas análises bivariadas, em que se considerou o valor de $\mathrm{p}<0,05$. 
Os adolescentes participaram da pesquisa de forma voluntária, após assinarem o Termo de Consentimento Livre e Esclarecido, sendo-Ihes assegurado o sigilo absoluto das informações, assim como a privacidade e o anonimato.

A pesquisa matricial foi submetida ao Comitê de Ética em Pesquisa do Hospital Universitário Júlio Muller, da Universidade Federal de Mato Grosso, e aprovada pelo protocolo n. 613 CEP-HUJM/2009, cumprindo, dessa forma, as normas que dispõem sobre Diretrizes e Normas Regulamentadoras de Pesquisas envolvendo seres humanos. O estudo foi aprovado pela Secretaria de Estado de Educação - SEDUC, bem como pelas diretorias das respectivas escolas.

\section{RESULTADOS}

De acordo com a Tabela 1, é possível observar que adolescentes do sexo masculino, em sua grande maioria (46,8\%), procuram um amigo para conversar quando o assunto é sexualidade. Já entre as meninas, assume valor mais expressivo as amigas (19,4\%), assim como os amigos $(17,1 \%)$ como fonte de diálogo, troca de experiências e esclarecimento de dúvidas. A afinidade com o mesmo sexo para tratar do assunto se confirma ao analisar que as mães são mais procuradas pelas meninas $(15,5 \%)$ e o pai, embora com valores discretos, exclusivamente pelos meninos $(p=0,0000)$.

Tabela 1. Distribuição dos adolescentes segundo o sexo, acerca de com quem se dá o diálogo sobre sexualidade. Cuiabá-MT, 2010

\begin{tabular}{|c|c|c|c|c|c|c|}
\hline \multirow{3}{*}{$\begin{array}{c}\text { COM QUEM SE DÁ O DIÁLOGO SOBRE } \\
\text { SEXUALIDADE }\end{array}$} & \multicolumn{4}{|c|}{ SEXO } & \multirow{2}{*}{\multicolumn{2}{|c|}{ TOTAL }} \\
\hline & \multicolumn{2}{|c|}{ Masculino } & \multicolumn{2}{|c|}{ Feminino } & & \\
\hline & $\mathbf{n}$ & $\%$ & $\mathbf{n}$ & $\%$ & $\mathbf{n}$ & $\%$ \\
\hline Amigo & 89 & 46,8 & 53 & 17,1 & 142 & 28,4 \\
\hline Namorado(a) & 13 & 6,8 & 11 & 3,5 & 24 & 4,8 \\
\hline Professor(a) & 3 & 1,6 & 1 & 0,3 & 4 & 0,8 \\
\hline Ninguém & 24 & 12,6 & 34 & 11,0 & 58 & 11,6 \\
\hline Outros & 5 & 2,6 & 5 & 1,6 & 10 & 2,0 \\
\hline Em branco & 1 & 0,5 & 3 & 1,0 & 4 & 0,8 \\
\hline Mais de uma & 28 & 14,7 & 78 & 25,2 & 106 & 21,2 \\
\hline Amiga & 4 & 2,1 & 60 & 19,4 & 64 & 12,8 \\
\hline Parente & 4 & 2,1 & 5 & 1,6 & 9 & 1,8 \\
\hline Enfermeiro(a) & 2 & 1,0 & 2 & 0,6 & 4 & 0,8 \\
\hline Irmão(a) & 1 & 0,5 & 7 & 2,3 & 8 & 1,6 \\
\hline Mãe & 7 & 3,7 & 48 & 15,5 & 55 & 11,0 \\
\hline Vizinho(a) & 3 & 1,6 & - & - & 3 & 0,6 \\
\hline Pai & 4 & 2,1 & - & - & 4 & 0,8 \\
\hline Médico & 1 & 0,5 & - & - & 1 & 0,2 \\
\hline Resposta nula & 1 & 0,5 & 2 & 0,6 & 3 & 0,6 \\
\hline TOTAL & 190 & 100,0 & 309 & 100,0 & 499 & 100,0 \\
\hline
\end{tabular}

$\mathrm{p}<0,001$.

O diálogo sobre sexualidade dentro do atual relacionamento é mais raro para as meninas, pois $30,4 \%$ nunca o tiveram com seus namorados / paqueras / ficantes; $27,5 \%$ sempre conversam sobre o assunto; $17,2 \%$ às vezes; $14,2 \%$ não responderam à pergunta; $10,0 \%$ referem ser rara essa conversa com o parceiro e $0,6 \%$ correspondem às respostas nulas. Já entre os meninos, $31,6 \%$ conversam às vezes com suas namoradas; $20,0 \%$ nunca conversam; $19,5 \%$ optaram pela 
resposta "sempre"; 15,8\% raramente conversam, $12,6 \%$ deixaram a pergunta em branco, e 0,5\% correspondem às respostas nulas, sendo o valor de $p<0,001$.

Em relação à idade com que tiveram sua primeira ejaculação, a maioria dos meninos $(49,7 \%)$ não se lembrava; 38,9\% referiram ter sido entre os 11 e 13 anos; 6,5\%, dos 8 aos 10 anos; 4,9\%, dos 14 aos 16 anos. Entre as meninas, 69,0\% referiram ter tido sua primeira menstruação dos 11 aos 13 anos; $12,1 \%$ não se lembravam; 11,8\%, dos 14 aos 16 anos; 6,7\%, dos 8 aos 10 anos; $0,3 \%$ corresponderam a respostas nulas, sendo o valor de $\mathrm{p}<0,001$.

No que diz respeito à iniciação sexual, entre as meninas, $67,1 \%$ ainda não haviam iniciado sua vida sexual; 30,6\% já haviam iniciado; e 2,3\% não responderam à pergunta. Já entre os meninos pesquisados, mais da metade $(55,0 \%)$ já haviam tido sua primeira relação; 41,3\% não se relacionavam sexualmente; e 3,7\% deixaram em branco $(p<0,001)$.

A faixa etária predominante em que as meninas tiveram sua primeira relação sexual foi dos 14 aos 16 anos (82,5\%), seguida pelas idades de 12 a 13 anos (12,0\%) e dos 17 aos 20 anos (5,5\%), com nenhum caso dos 8 aos 10 anos. Pouco mais da metade dos meninos (51,3\%) também teve sua primeira relação entre 14 e 16 anos; 39,1\%, dos 12 aos 13 anos; 7,3\%, dos 8 aos 10 anos; e 2,4\%, dos 17 aos 20 anos ( $<<0,001)$.

Analisando-se apenas os adolescentes que já tiveram sua primeira relação sexual, a Tabela 2 mostra que, de um modo geral, a primeira relação sexual das adolescentes em questão foi com namorado(a); esse valor assume $86,2 \%$ do total de meninas, porém, entre os meninos, 30,8\% iniciou a vida sexual com uma paquera e apenas $24,0 \%$ com namorada(o).

Tabela 2. Distribuição dos adolescentes de acordo com o sexo, a respeito de com quem tiveram sua primeira relação sexual. Cuiabá-MT, 2010

\begin{tabular}{|c|c|c|c|c|c|c|}
\hline \multirow{3}{*}{ PRIMEIRO PARCEIRO(A) SEXUAL } & \multicolumn{4}{|c|}{ SEXO } & \multirow{2}{*}{\multicolumn{2}{|c|}{ TOTAL }} \\
\hline & \multicolumn{2}{|c|}{ Masculino } & \multicolumn{2}{|c|}{ Feminino } & & \\
\hline & $\mathbf{n}$ & $\%$ & $\mathbf{n}$ & $\%$ & $\mathbf{n}$ & $\%$ \\
\hline Namorado(a) & 25 & 24,0 & 81 & 86,2 & 106 & 53,5 \\
\hline Paquera & 32 & 30,8 & 8 & 8,5 & 40 & 20,2 \\
\hline Conhecido(a) & 16 & 15,4 & 1 & 1,1 & 17 & 8,6 \\
\hline Desconhecido(a) & 6 & 5,8 & - & - & 6 & 3 \\
\hline Amigo(a) & 11 & 10,6 & 2 & 2,1 & 13 & 6,6 \\
\hline Parente & 5 & 4,8 & - & - & 5 & 2,5 \\
\hline Não lembro & 1 & 1,0 & - & - & 1 & 0,5 \\
\hline Outro & 3 & 2,9 & 1 & 1,1 & 4 & 2 \\
\hline Em branco & 5 & 4,8 & 1 & 1,1 & 6 & 3 \\
\hline TOTAL & 104 & 100,0 & 94 & 100,0 & 198 & 100,0 \\
\hline
\end{tabular}

$p=0,001$.

A primeira relação sexual dos adolescentes do sexo masculino foi para $74,0 \%$ uma decisão tomada em comum acordo; $10,6 \%$ referiram ter pressionado; 9,6\% foram pressionados; e 5,8\% não responderam. Entre as meninas, $89,4 \%$ delas, referiram ter sido uma decisão em comum acordo; $4,3 \%$ foram pressionadas; 3,2\% pressionaram; e 3,2\% deixaram a resposta em branco, sendo o valor de $p=0,0468$.
A pesquisa revelou que, dos adolescentes do sexo masculino que já tiveram sua iniciação sexual, 69,3\% fizeram uso de algum método para evitar gravidez, DST / AIDS; 24,0\% não os utilizaram; e 6,7\% não responderam à pergunta. Entre as meninas já iniciadas sexualmente, $89,2 \%$ fizeram uso de algum método; $8,6 \%$ não fizeram; e $2,2 \%$ deixaram a resposta em branco, sendo o valor de $p=0,0028$. 
Entre os que fizeram uso de método contraceptivo, 92,9\% referiram ter sido o preservativo; 4,5\% referiram ter utilizado o anticoncepcional oral; $1,9 \%$, a injeção; e $0,7 \%$ não responderam. Importante salientar que, entre os $4,5 \%$ que responderam fazer uso de anticoncepcional oral, a metade, além da pílula, fez uso de preservativo conjuntamente.

A maioria dos adolescentes $(67,2 \%)$ possuía vida sexual ativa no momento da coleta de dados, sendo que essa proporção era de $66,5 \%$ entre os meninos e de $71,3 \%$ entre as meninas. Vale ressaltar que o valor de " $\mathrm{p}$ " está acima de 5\%, ou seja, as diferenças entre os sexos não são estatisticamente significativas ( $p=0,1773)$.

Quando questionados quanto à frequência com que praticam sexo, a maioria dos adolescentes referiu ter relações sexuais às vezes $(33,8 \%) ; 23,2 \%$, semanalmente; $13,6 \%$, raramente; $10,1 \%$, diaria- mente; $8,1 \%$, quinzenalmente; $7,1 \%$ não responderam à pergunta; e 4,0\%, mensalmente. Constatou-se que a diferença entre os sexos não foi estatisticamente significativa, uma vez que o valor de " $\mathrm{p}$ " apresentou-se bem acima do esperado ( $p=0,7928)$.

Quanto à opção sexual, a grande maioria dos adolescentes envolvidos na pesquisa $(71,7 \%)$ referiu ser heterossexual; $21,2 \%$ deixaram a resposta em branco; 3,5\% marcaram a opção homossexual; e 3,5\% se declararam bissexuais (3,5\%). Esse dado não tem valor estatístico em relação à diferenciação por sexo, uma vez que o valor foi de $p=0,7007$.

A análise da Tabela 3 permite inferir que, entre as meninas, $72,3 \%$ tiveram de 1 a 3 parceiros sexuais nos últimos três meses. Já entre os meninos, quase metade $(48,1 \%)$ não se lembrava do número de parceiras sexuais que haviam tido nos últimos três meses.

Tabela 3. Distribuição dos adolescentes segundo o sexo, acerca do número de parceiros sexuais nos últimos três meses. Cuiabá-MT, 2010

\begin{tabular}{|c|c|c|c|c|c|c|}
\hline \multirow{3}{*}{ NÚMERO DE PARCEIROS SEXUAIS } & \multicolumn{6}{|c|}{ SEXO } \\
\hline & \multicolumn{2}{|c|}{ Masculino } & \multicolumn{2}{|c|}{ Feminino } & \multicolumn{2}{|c|}{ TOTAL } \\
\hline & $\mathbf{n}$ & $\%$ & $\mathbf{n}$ & $\%$ & $\mathbf{n}$ & $\%$ \\
\hline 1 a 3 & 20 & 19,2 & 68 & 72,3 & 88 & 44,4 \\
\hline 4 a 6 & 7 & 6,7 & 10 & 10,6 & 17 & 8,6 \\
\hline 7 a 10 & 3 & 2,9 & - & - & 3 & 1,5 \\
\hline Mais de 10 & 3 & 2,9 & - & - & 3 & 1,5 \\
\hline Não lembro & 50 & 48,1 & 9 & 9,6 & 59 & 29,8 \\
\hline Em branco & 21 & 20,2 & 7 & 7,4 & 28 & 14,1 \\
\hline TOTAL & 104 & 100,0 & 94 & 100,0 & 198 & 100,0 \\
\hline
\end{tabular}

$p<0,001$.

De acordo com a Tabela 4, entre os que declararam vida sexual ativa no momento da coleta dos dados, uma porcentagem maior das meninas $(68,0 \%)$ sempre usa preservativo em suas relações, quando comparada com os meninos
$(56,7 \%)$. De forma geral, mais da metade dos adolescentes $(62,1 \%)$ referem usar preservativo sempre. Vale ressaltar a presença de adolescentes que nunca usam o preservativo $(5,0 \%)$ e os que o utilizam apenas às vezes $(25,2 \%)$.

Tabela 4. Distribuição dos adolescentes segundo o sexo, em relação à frequência com que usam preservativo em suas relações sexuais. Cuiabá-MT, 2010

\begin{tabular}{|c|c|c|c|c|c|c|}
\hline \multirow{3}{*}{ QUANDO USA CAMISINHA } & \multicolumn{6}{|c|}{ SEXO } \\
\hline & \multicolumn{2}{|c|}{ Masculino } & \multicolumn{2}{|c|}{ Feminino } & \multicolumn{2}{|c|}{ TOTAL } \\
\hline & $\mathbf{n}$ & $\%$ & $\mathbf{n}$ & $\%$ & $\mathbf{n}$ & $\%$ \\
\hline Sempre & 59 & 56,7 & 64 & 68,0 & 123 & 62,1 \\
\hline Às vezes & 30 & 28,8 & 20 & 21,3 & 50 & 25,2 \\
\hline Nunca & 3 & 2,9 & 7 & 7,4 & 10 & 5,0 \\
\hline Em branco & 12 & 11,5 & 2 & 2,1 & 14 & 7,0 \\
\hline Resposta nula & - & - & 1 & 1,0 & 1 & 0,5 \\
\hline TOTAL & 104 & 100,0 & 94 & 100,0 & 198 & 100,0 \\
\hline
\end{tabular}

$p=0,0157$. 


\section{DISCUSSÃO}

O fato de a fonte de diálogo sobre sexualidade ser, predominantemente, os amigos entre os meninos e amigas entre as meninas (com dependência estatística entre as variáveis), pode estar relacionado com a dificuldade que pais e professores têm para abordar essa temática no dia a dia, não permitindo, com isso, que os jovens tenham abertura com a família ou na escola para esclarecerem suas dúvidas ${ }^{6}$.

É preciso destacar que, diante do silêncio em casa, o adolescente tende a procurar informações com outros adolescentes também imaturos, contribuindo, dessa maneira, para a prática sexual de forma insegura ${ }^{7}$.

Alguns autores, nesse sentido, destacam a importância dos pais como os primeiros educadores sexuais, pois a eles compete a maior responsabilidade na formação dos seus filhos, representando modelos que contribuem para a formação de sua identidade sexual ${ }^{7}$. Entretanto, estudos realizados revelam que alguns pais não se sentem à vontade para falar sobre a sexualidade com os filhos e receiam não ter a informação apropriada que corresponda às necessidades, nos diferentes níveis etários ${ }^{7}$. Outros são da opinião de que se falarem com os filhos sobre sexualidade promoverão o início da sua atividade sexual mais cedo que o esperado ${ }^{8}$. Contudo, em uma pesquisa realizada no ano de 2008, com 109 sujeitos (adolescentes, pais e professores), pertencentes à Escola Básica Francisco de Arruda, de Lisboa-Portugal, com intuito de analisar a influência da família e da escola na educação sexual dos adolescentes, observou que os adolescentes que possuem práticas sexuais com prevenção eram os que tinham uma melhor comunicação com os seus progenitores ${ }^{8}$.

Apesar da dificuldade dos pais, é no convívio familiar, entre pessoas que se estimam e tentam superar as dificuldades do dia a dia, que as questões de sexualidade devem ser debatidas, levando-se em conta os valores, atitudes, crenças religiosas e culturais da família ${ }^{6}$.

Convicções errôneas, ideias falsas, escrúpulos sem fundamento positivo e a falta de diálogo em casa sobre sexualidade podem desencadear consequências irreversíveis, como a gravidez precoce, e favorecer condições de risco para o adolescente, como $\mathrm{DST}^{7}$.

A falta de diálogo sobre sexo dentro do atual relacionamento, principalmente entre as meninas (com dependência estatística entre as variáveis), é apontada por muitos autores como preocupante, pois o diálogo dentro do relacionamento, em especial no que diz respeito à contracepção / proteção, interfere positivamente no uso do preservativo, como indica pesquisa em que as moças e os rapazes que conversaram sobre métodos contraceptivos / proteção antes da iniciação utilizaram mais preservativo nessa relação ${ }^{9}$.

A maior parte dos meninos envolvidos na presente pesquisa não se lembrava com que idade teve sua primeira ejaculação. Já a menarca é considerada como um evento marcante para as meninas, pois grande parte delas soube referir quando esse evento ocorreu. Destaca-se a dependência estatística entre as variáveis.

Apesar de a menarca ser considerada um período marcante para as meninas por caracterizar o início da vida reprodutiva e envolver grandes transformações, alguns autores questionam se é um indicador de maturação adequado, pois, apesar de acontecer em uma determinada idade, uma garota somente seria considerada sexualmente madura quando o ciclo menstrual se tornasse regular ${ }^{10}$.

O fato de a grande parte das meninas ainda não ter iniciado sua vida sexual, ao mesmo tempo em que mais da metade dos meninos já o terem feito (com dependência estatística entre as variáveis), nos remete à questão cultural e de gênero que permeia todo o processo da sexualidade humana. Nesse sentido, por uma questão cultural da sociedade em que vivemos, aos homens cabe o papel, culturalmente imposto, de não resistir ao impulso sexual, enquanto às muIheres cabe o papel de controlar seus impulsos, ou, ao menos, cedê-los apenas às pessoas com as quais têm vínculos afetivo-amorosos, ratificando as relações de gênero presentes no cenário da iniciação sexual e que tem estimulado os jovens do sexo masculino a iniciarem suas práticas sexuais bem mais cedo do que o sexo feminino ${ }^{11,12}$.

Quanto à iniciação sexual em idades precoces (14 aos 16 anos), tanto entre meninas quanto meninos (com dependência estatística entre as 
variáveis), sugere-se que possa haver, muito possivelmente, um código de conduta prescrevendo que, em determinado momento ou a partir de certa idade, a virgindade passa a ser um peso na vida dessas adolescentes, tal como tem ocorrido entre os homens há tempos, servindo como elemento de pressão para que haja a iniciação sexual ${ }^{13}$. A autora ainda destaca que algumas adolescentes iniciam a vida sexual porque se sentem pressionadas pelos seus parceiros, embora no presente estudo grande parte dos adolescentes referiu que a primeira relação foi uma decisão tomada em comum acordo. Sendo assim, a iniciação sexual em idades cada vez menores é analisada por estudiosos como uma evidência das transformações ocorridas no comportamento sexual da população brasileira, por conta do uso generalizado de métodos contraceptivos e de prevenção, permitindo a desvinculação do ato sexual à reprodução e liberando-os do medo da AIDS ${ }^{12}$.

$O$ fato de a grande maioria das meninas ter tido sua primeira relação sexual com namorado(a), enquanto os meninos tiveram sua primeira experiência sexual com ficante / paquera (com dependência estatística entre as variáveis), reforça a hipótese de que a atividade sexual para as mulheres relaciona-se com envolvimento emocional. Há que se destacar que os relacionamentos na adolescência são caracterizados, atualmente, não apenas pelo namoro, mas também pelo "ficar", em que são abertos caminhos para a mútua exploração sexual ${ }^{11}$.

A baixa adesão ao uso de métodos para evitar DST / AIDS e gravidez é analisada por alguns autores como o oposto da espontaneidade que se costuma atribuir ao sexo e à juventude ${ }^{5}$. Os adolescentes apontam numerosas justificativas para o não uso do preservativo: esquecimento, custos e desprazer na relação sexual. Assim, o estímulo oferecido para utilização desse método deve incluir a dimensão do erotismo e da praticidade, não apenas o medo. Os adolescentes, em geral, sabem que o preservativo evita doenças e gravidez, mas, mesmo assim, não o usam, pois existe uma enorme lacuna entre o nível de conhecimento e seu uso efetivo.

Vale destacar que se percebe entre as meninas uma preocupação maior no que diz res- peito à prevenção (com dependência estatística entre as variáveis). Uma possível justificativa para o menor uso de métodos de prevenção pelos meninos trata-se do tabu ainda existente em torno do preservativo, em que o prazer masculino tem redução com seu uso, acarretando em resistência por parte dos adolescentes do sexo masculino $^{14}$.

Quanto ao número de parceiros sexuais nos últimos três meses verificado na presente casuística (com dependência estatística entre as variáveis), em uma pesquisa realizada no ano de 2008, em Concórdia-SC, com o objetivo de investigar a iniciação sexual de adolescentes do sexo masculino ${ }^{3}$, observou que $32,5 \%$ dos adolescentes do sexo masculino tem relações sexuais com parceiras(os) eventuais encontradas(os) em bares, festas, bailes, bem como prostitutas, o que evidencia a variação de parceria sexual e a vulnerabilidade, nos remetendo à ideia de que boa parte desses adolescentes mantém uma certa frequência de relações sexuais, mas não com parceiras fixas, o que coincide com a ocasionalidade das relações relatadas no presente estudo, bem como o despreparo no que diz respeito à prevenção.

Os autores citados acima ainda reforçam que há uma forte relação de gênero nessa questão, pois o gostar da parceira e a parceria fixa (namorada e esposa) sugerem valorização do envolvimento emocional em uma relação sexual, o que tradicionalmente é considerada característica apenas da sexualidade feminina.

Quanto à opção sexual (sem dependência estatística entre as variáveis), a presença da homossexualidade nos remete a algumas reflexões. Entre elas, a rejeição e a discriminação existentes no meio social, o que acarreta em dificuldade para assumi-la. A orientação sexual de alguém ainda é um processo não totalmente conhecido pela ciência. Vários estudos já foram realizados nesse sentido, mas ainda são apenas hipóteses ${ }^{15}$. Sendo a adolescência uma etapa em que as manifestações sexuais se intensificam e a identidade sexual se reafirma, é comum surgirem dúvidas / incertezas quanto ao tema. Aqueles que sentem atração por alguém do mesmo sexo, inicialmente, sentem-se diferentes, sem saber o porquê ${ }^{15}$. A 
consciência do desejo sexual acontece progressivamente. Geralmente, os homossexuais descobrem sua inclinação sexual no início da adolescência, têm fantasias homoeróticas, passam a ter experiências sexuais e assumem a sua homossexualidade no início da vida adulta. O intervalo de tempo entre descobrir-se homossexual e revelar-se pode ser longo. A maioria dos adolescentes tende a se manifestar quando já se considera independente e sente-se mais seguro em relação à sua orientação sexual. Alguns não se revelam nunca.

O que é preciso destacar, nesse sentido, é a dificuldade dos adolescentes em buscarem o serviço de saúde em razão dos problemas relacionados às suas experiências homossexuais ${ }^{16}$. Portanto, a orientação e acompanhamento da equipe de saúde tornam-se imprescindíveis para abrir espaços e dar subsídios para esses adolescentes que lidam com o "diferente".

$\mathrm{O}$ uso do preservativo mais frequente entre as meninas (com dependência estatística entre as variáveis) coincide com a discussão de alguns autores ${ }^{9}$ que sinalizam essa diferença de gênero no que concerne às concepções e preocupações vinculadas aos relacionamentos afetivo-sexuais. Vale ressaltar também que as meninas naturalmente assumem uma postura mais madura e responsável primeiro que os meninos.

Uma das possíveis causas para o não uso do preservativo em uma porcentagem ainda significativa da população adolescente consiste no fato de estarem passando por uma fase em que há indefinição da identidade sexual, com experimentação e variabilidade de parceiros, fazendo com que se sintam invulneráveis, se expondo a riscos sem prever suas consequências ${ }^{17}$.

Apesar desse comportamento vulnerável, uma pesquisa realizada com jovens de 18 a 24 anos de ambos os sexos buscou identificar quais os fatores relacionados ao uso do preservativo pelos jovens nas capitais: Porto Alegre-RS, Rio de Janeiro-RJ e Salvador-BA, no ano de 2002. Constatou-se que os jovens que usam preservativo na iniciação sexual tendem a manter essa prática no decorrer de sua vida, o que reforça a necessidade de orientação continuada para o estímulo ao uso do preservativo ${ }^{9}$.

\section{CONCLUSÃO}

Os resultados revelam a existência de comportamento de risco entre adolescentes no que se refere à sexualidade, o que confirma a hipótese da pesquisa.

Com base no exposto, pôde-se concluir que o diálogo acerca da sexualidade ainda representa uma barreira a ser superada não só pelos adolescentes, mas também, e principalmente, pela família, que ainda carrega tabus para tratar do assunto. Dessa forma, a participação dos pais na vida sexual dos filhos pode contribuir para práticas sexuais mais seguras, em se tratando de prevenção. É possível, também, criar ambientes favoráveis na escola para discussão do tema nesse grupo etário, muitas vezes denominado vulnerável, contribuindo para a prática sexual na adolescência de forma saudável e segura.

A expressividade do número de parceiros(as) sexuais entre os adolescentes merece especial atenção, pois demanda constante uso de medidas de prevenção contra DST / AIDS.

No tocante à questão de gênero, é perceptível o menor comprometimento dos meninos quando comparado às meninas, revelado não só pela menor adesão ao uso do preservativo, como também à percepção da prática sexual livre de compromisso, uma vez que a maior parte dos meninos envolvidos na pesquisa teve sua primeira relação sexual fora de um relacionamento estável, o que, geralmente, não acontece com as meninas.

Frente à complexidade e fatores de risco que envolvem a sexualidade na adolescência, torna-se oportuno o desenvolvimento de estratégias e políticas que privilegiem esse grupo, além de estudos que possam aprofundar o conhecimento na área. 


\section{REFERÊNCIAS}

1. Soares SM, Amaral MA, Silva LB, Silva PAB, et al. Oficinas sobre sexualidade na adolescência: revelando vozes, desvelando olhares de estudantes do ensino médio. Esc Anna Nery Rev Enferm. 2008;12(3):485-91.

2. Tomita TY, Ferrari RAP. Adolescência e sexualidade no cotidiano da equipe de enfermagem do serviço de atenção básica de saúde. Semina Ciên Biol Saúde. 2007;28(1):39-52.

3. Gubert D, Madureira VSF. Iniciação sexual de homens adolescentes. Ciên Saúde Colet. 2008;13(2):2247-56.

4. DATASUS. Morbidade. Cuiabá; 2009 [acesso 17 Mar 2010]. Disponível em: http://tabnet.datasus.gov.br/cgi/tabcgi. exe?sih/cnv/nruf.def

5. Paiva V, Calazans G, Venturi G, Dias R. Idade e uso de preservativo na iniciação sexual de adolescentes brasileiros. Rev Saúde Pública. 2008;42(Supl 1):45-53.

6. Alves AS, Lopes MHBM. Uso de métodos anticoncepcionais entre adolescentes universitários. Rev Bras Enferm. 2008;61(2):170-7.

7. Alves CA, Brandão ER. Vulnerabilidades no uso de métodos contraceptivos entre adolescentes e jovens: interseções entre políticas públicas e atenção à saúde. Ciênc Saúde Coletiva 2009;14(2):661-70.

8. Janeiro JMSV. Educar sexualmente os adolescentes: uma finalidade da família e da escola? Rev Gaúcha Enferm. 2008;29(3):382-90.

9. Teixeira AMFB, Knauth DR, Fachel JMG, Leal AF, et al. Adolescentes e uso de preservativos: as escolhas dos jovens de três capitais brasileiras na iniciação e na última relação sexual. Cad Saúde Pública. 2006;22(7):1385-96.

10. Klug DP, Fonseca PHS. Análise da maturação feminina: um enfoque na idade de ocorrência da menarca. Rev Educação Física UEM. 2008;17(2):139-47.

11. Borges ALV. Relações de gênero e iniciação sexual de mulheres adolescentes. Rev Esc Enferm USP. 2007;41(4):597604.

12. Pelloso SM, Carvalho MDB, Higarashi IH. Sexualidade e gênero: um estudo com adolescentes em um município de pequeno porte do Noroeste do Paraná. Acta Sci Health Sci. 2008;30(2):113-9.

13. Borges ALV. Pressão social do grupo de pares na iniciação sexual de adolescentes. Rev Esc Enferm USP. 2007;41(n. e.):782-6.

14. Asinelli-Luz A, Fernandes Jr N. Gênero, adolescências e prevenção ao HIV/AIDS. Pró-Posições. 2008;19(2):81-97.

15. Filho FST, Marretto CAR. Apontamentos sobre o atentar contra a própria vida, homofobia e adolescências. Rev Psicol UNESP. 2008;7(1):133-51.

16. Oliveira TC, Carvalho LP, Silva MA. O enfermeiro na atenção à saúde sexual e reprodutiva dos adolescentes. Rev Bras Enferm. 2008;61(3):306-11.

17. Custódio G, Schuelter-Trevisol F, Trevisol DJ, Zappelini CEM, et al. Comportamento sexual e fatores de risco para a ocorrência de gravidez, DST e HIV em estudantes do município de Ascurra (SC). Arq Catarinenses Med. 2009;38(1):56-61.

Recebido em: 22 de maio de 2013. Versão atualizada em: 1 de agosto de 2013. Aprovado em: 28 de agosto de 2013. 